\title{
Fractal Functions in Interpolation and Approximation: A Bird's-Eye View
}

\author{
Navascues $\mathrm{MA}^{1 *}$, Chand $\mathrm{AKB}^{2}$, Viswanathan $\mathrm{P}^{3}$ and Sebastan $\mathrm{MV}^{4}$
}

${ }^{1}$ Departmento de Matem'atica Aplicada, Escuela de Ingenier'ia y Arquitectura, Universidad de Zaragoza, Spain

${ }^{2}$ Department of Mathematics, Indian Institute of Technology Madras, Chennai-600036, India

${ }^{3}$ Mathematical Sciences Institute, Australian National University, Australia

${ }^{4}$ Centro Universitario de la Defensa de Zaragoza Academia General Militar, Spain

\begin{abstract}
This article is targeted to provide a brief and coarse discussion on theory of fractal functions and their recent developments including some of the researches of the authors. Materials on fractal functions provided by this paper is not claimed to be exhaustive, but is intended to be read before or in parallel with technical papers available in the literature on this subject. We have made an earnest attempt to supply an overall flavor of the subject of univarite fractal approximation and useful source of links to assist a novice and perhaps to an expert in fractal functions too.
\end{abstract}

\section{Prologue}

Construction of a continuous function from given discrete data, which is referred to as interpolation, has been applied in various fields since time immemorial. In classical Numerical Analysis and Approximation Theory, there are several interpolation methods (for instance, polynomial, trigonometric, spline, rational) that can be applied to a specific data set, according to the assumptions that underlie the model to be investigated. Undoubtedly, these traditional (non-fractal) nonrecursive techniques constitute a great tool to model various physical phenomena. However, these methods produce interpolants with Hausdorff dimension one and hence if a given data set is more complex and irregular (for instance, data sampled from real-world signals such as financial series, seismic data, and bioelectric recordings), then they may not provide satisfactory results. Consequently, to model these signals, need for interpolants that are nondifferentiable in dense set of points in the domain arose quite naturally. About a quarter century has passed since Barnsley [1] introduced Fractal Interpolation Function (FIF), a concept which soon turned out to be the right frame for a constructive approximation theory of non-differentiable functions, by using the notion of Iterated Function System (IFS). In view of their diverse applications, there has been steadily increasing interest in the theory of fractal functions, and it still continues to be a hot topic of research. Following the publication of Fractals Everywhere [2], a beautiful exposition of IFS theory, fractal functions and their applications, various related issues such as calculus, Holder continuity, convergence, stability, smoothness, determination of scaling parameters, and perturbation error have been investigated in the literature [3-13]. The concept of smooth FIFs has been used to generalize the traditional splines [14-18] and to demonstrate that the interaction of classical numerical methods with fractal theory provides new interpolation schemes that supplement the existing ones. Various other extensions of FIFs include multivariable FIFs [7,9,16,19-25] generated by using higher-dimensional or recurrent IFSs, the hidden variable FIFs produced by projecting the attractors of vector valued IFSs to a lower-dimensional space.

The notion ofFIFs can be used to associate a family of fractal functions $f^{\alpha}$ parameterized by a suitable vector $\alpha$ with a prescribed continuous function $\mathrm{f}$ on a compact interval I. Using this "fractal perturbation", Navascues introduced the operator $F^{\alpha}: c(\mathrm{I}) \rightarrow c(\mathrm{I})$ via $F^{\alpha}(\mathrm{f})=\mathrm{f}^{\alpha}$ and its extension to more general spaces $L^{p}(\mathrm{I})$ using standard density arguments. These maps tend to bridge the gap between smoothness of classical mathematical objets and pseudo-randomness of experimental variables, breaking in this way their apparent diversity. Navascues and coworkers $[17,18,22]$ contributed to the theory by defining "rough" approximants as perturbation of the functions generally used in classical approximation (polynomial, trigonometric, rational, etc.) via this operator. Recently [23], the authors investigated conditions on the elements of the IFS so that the perturbation preserves the basic shape properties inherent in the original function, thereby paving a way to shape preserving fractal approximation, an interesting area that needs further exploration at least in our opinion.

\section{A Brief Outline of Fractal Interpolation Function}

By fractal function, we mean basically a function whose graph is the attractor of an IFS, which was first constructed as an interpolant as follows. Consider a set of data points $\left\{\left(x_{n}, y_{n}\right) \in \mathbb{R}^{2}: n=1,2, \ldots, N\right\}$ with increasing abscissae. Set $I=\left[x_{1}, x_{N}\right]=\{1,2, \ldots, \mathrm{N}-1\}$. For $i \in \mathbb{N}_{N-1}$, set $I_{i}=\left[\mathrm{x}_{\mathrm{i}}, \mathrm{x}_{\mathrm{i}+1}\right]$ and let $L_{i}: I \rightarrow I_{i}$ be affine maps such that $L_{i}\left(\mathrm{x}_{1}\right)=\mathrm{x}_{\mathrm{i}}, L_{i}\left(\mathrm{x}_{\mathrm{N}}\right)=\mathrm{x}_{\mathrm{i}+1}$ Let $K=I \times[\mathrm{c}, \mathrm{d}],-\infty<c<d<+\infty$ Consider $\mathrm{N}-1$ continuous maps $F_{i}: \mathrm{K} \rightarrow[\mathrm{c}, \mathrm{d}]$ satisfying

$$
\left|F_{i}(\mathrm{x}, \mathrm{y})-\mathrm{F}_{\mathrm{i}}\left(\mathrm{x}, \mathrm{y}^{*}\right)\right| \leq \mathrm{r}_{\mathrm{i}}\left|y-y^{*}\right| ; \mathrm{F}_{\mathrm{i}}\left(\mathrm{x}_{1}, \mathrm{y}_{1}\right)=\mathrm{y}_{\mathrm{i}}, F_{i}\left(\mathrm{x}_{\mathrm{N}}, \mathrm{y}_{\mathrm{N}}\right)=\mathrm{y}_{\mathrm{i}}+1
$$

Where $x \in I, y, y^{*} \in[\mathrm{c}, \mathrm{d}]$, and $0 \leq r_{i}<1$. Now define

$$
W_{i}: K \rightarrow I_{i} \times[\mathrm{c}, \mathrm{d}] \subseteq \mathrm{K}, \mathrm{W}_{\mathrm{i}}(\mathrm{x}, \mathrm{y}):=\left(\mathrm{L}_{\mathrm{i}}(\mathrm{x}), \mathrm{F}_{\mathrm{i}}(\mathrm{x}, \mathrm{y})\right) \forall \mathrm{i} \in \mathbb{N}_{\mathrm{N}-1}
$$

For the collection $I^{*}:=\left\{K ; W_{i}: i \in \mathbb{N}_{N-1}\right\}$, which is termed IFS, Barnsley presented the following seminal result.

Theorem 1 (Barnsley [1]) Corresponding to the IFS $I^{*}=\left\{\mathrm{K} ; \mathrm{W}_{\mathrm{i}}: \mathrm{i} \in \mathbb{N}_{\mathrm{N}-1}\right\}$ there exists a unique set $\mathrm{G}$ satisfying

$G=U_{i \in \mathbb{N} N-1} W_{i}(\mathrm{G})$ and $\mathrm{G}$ is the graph of a continuous function $g: I \rightarrow \mathbb{R}$ satisfying $\mathrm{g}(\mathrm{xn})=\mathrm{yn}$ for $\mathrm{n}=1,2, \ldots \mathrm{N}$. Let

*Corresponding author: Navascues MA, Departmento de Matem'atica Aplicada Escuela de Ingenier'ıa y Arquitectura, Universidad de Zaragoza, Spain, Tel: 34976761000; E-mail: manavas@unizar.es

Received October 24, 2014; Accepted October 25, 2014; Published October 31 2014

Citation: Navascues MA, Chand AKB, Viswanathan P, Sebastan MV (2014) Fractal Functions in Interpolation and Approximation: A Bird's-Eye View. J Appl Computat Math 3: 188. doi:10.4172/2168-9679.1000188

Copyright: ( 2014 Navascues MA, et al. This is an open-access article distributed under the terms of the Creative Commons Attribution License, which permits unrestricted use, distribution, and reproduction in any medium, provided the original author and source are credited. 
$G:=\left\{\mathrm{g}^{*} \in \mathrm{c}(\mathrm{I}): \mathrm{g}^{*}\left(\mathrm{x}_{1}\right)=\mathrm{y}_{1}, \mathrm{~g}^{*}\left(\mathrm{x}_{\mathrm{N}}\right)=\mathrm{y}_{\mathrm{N}}\right\}$ be endowed with the uniform metric. If $T: G \rightarrow G,\left(\mathrm{Tg}^{*}\right)$

$(\mathrm{x}):=\mathrm{F}_{\mathrm{i}}\left(\mathrm{L}_{i}^{-1}(\mathrm{x}), \mathrm{g}^{*} \mathrm{oL}_{i}^{-1}(\mathrm{x})\right), \mathrm{x} \in \mathrm{I}_{\mathrm{i}}, i \in \mathbb{N}_{N-1}$ then $\mathrm{g}$ is the unique xed point of $\mathrm{T}$.

Definition 1 The function $g$ appearing in the previous theorem is called a Fractal Interpolation

Function (FIF) and it fulfills the functional equation $g\left(\mathrm{~L}_{\mathrm{i}}(\mathrm{x})\right)=\mathrm{F}_{\mathrm{i}}(\mathrm{x}, \mathrm{g}(\mathrm{x}))$ for $x \in I$ and $i \in \mathbb{N}_{N-1}$.

Most widely studied fractal functions in the theory and applications heretofore are defined by the

IFS $I^{*}=\left\{\mathrm{K} ;\left(\mathrm{L}_{\mathrm{i}}(\mathrm{x}), \mathrm{F}_{\mathrm{i}}(\mathrm{x}, \mathrm{y})\right): \mathrm{i} \in J\right\}$ with constituent maps

$$
L_{i}(\mathrm{x})=\mathrm{a}_{\mathrm{i}} x+b_{i}, F_{i}(\mathrm{x}, \mathrm{y})=\alpha_{\mathrm{i}} y+q i(\mathrm{x}), \mathrm{i} \in \mathbb{N}_{\mathrm{N}-1}
$$

Where $\left|\alpha_{\mathrm{i}}\right|<1$ and qi are suitable continuous functions so that the maps Fi satisfy conditions in (1). A possible explanation for the choice of this special class of IFS is that the corresponding FIF is explicitly integrable, a satisfactory theory for moment integrals can be developed and it is easy to discover functional equations obeyed by Fourier and Laplace transforms. Following Theorem 1, the FIF g can be constructed recursively using the functional equation

$$
g\left(\mathrm{~L}_{\mathrm{i}}(\mathrm{x})\right)=\mathrm{F}_{\mathrm{i}}(\mathrm{x}, \mathrm{g}(\mathrm{x}))=\alpha_{\mathrm{i}} g(\mathrm{x})+\mathrm{q}_{\mathrm{i}}(\mathrm{x}), \mathrm{x} \in \mathrm{I}, \mathrm{i} \in \mathbb{N}_{\mathrm{N}-1}
$$

In what follows, we shall get into some specifics of particular flavors of fractal interpolation for the benefit of the reader, specifically for one who wants to critically compare the FIF scheme with schemes available in the traditional Numerical Analysis. It is plain to see that the presence of free parameters $i$, which determine Hausdorff dimension of the graph of the interpolant, provides additional flexibility to the choice of particular function in contrast to the unicity inherent in a specific kind of traditional interpolation. However, finding an "optimal" scale vector is a quite challenging problem for which different approaches are discussed in the literature. Note that apart from this recursive functional equation, the FIF g possesses an explicit representation in terms of an infinite series [11]. Further, g can be expressed using the technique of operator approximation, see [13]. As with wavelets and many other new function types, "closed form" expressions for FIF generally take the form of one of the two types of algorithms, chaos game (a Markov chain Monte Carlo algorithm) and deterministic iteration; both approaches are highly accurate and have been reported in many places in the literature $[5,6]$. In many cases, evaluation of a FIF at a specific point can be achieved by summing a rapidly convergent series. The functional equation for the FIF provides a rule to predict the values of the interplant at refined mesh points, and thus may remind a subdivision scheme available in the traditional Numerical Analysis. Let us note here that a FIF, in fact, provides a subdivision scheme, and the fact that it arises from an IFS makes the mathematical treatments such as convergence, smoothness, etc. of those subdivision schemes relatively easier to handle. Most of the traditional interpolants have locality, whereas particular feature of the fractal methods is that they explore the self-similarity and are global, in general. The benefit is that subtle global dependencies reflect nature and may lead to harmonious and attractive non-cookie cutter forms, shapes and approximants. The same is true for Bezier curves, much used in computer graphics and design packages. It should be borne in mind that for particular values of the parameters, the fractal interpolation schemes can recapture local traditional schemes.

\section{Fractal Functions in Approximation and Fractal Operator}

Reviving the spirit of the previous section, let $f \in c(\mathrm{I})$ Consider (2) with $q_{i}(\mathrm{x})=$ f o $L_{i}(\mathrm{x})-\alpha_{\mathrm{i}} b(\mathrm{x})$,

$\mathrm{x} \in \mathrm{I}, \mathrm{b}: \mathrm{I} \rightarrow \mathbb{R}$ is a continuous function, $\mathrm{b}(\mathrm{x} 1)=\mathrm{f}(\mathrm{x} 1), \mathrm{b}(\mathrm{xN})=$ $\mathrm{f}(\mathrm{xN})$, and $\mathrm{b} \neq \mathrm{f}$. In view of (3), corresponding fractal function $f^{\alpha}$ satisfies.

$$
f^{\alpha}(\mathrm{x})=\mathrm{f}(\mathrm{x})+\alpha_{\mathrm{i}}\left(\mathrm{f}^{\alpha}-\mathrm{b}\right) \mathrm{oL}_{i}^{-1}(\mathrm{x}) \forall \mathrm{x} \in \mathrm{I}_{\mathrm{i}}, i \in \mathbb{N}_{N-1}
$$

Notice that if $\alpha:=0 \in \mathbb{R}^{N-1}$ then $f^{\alpha}=f$ and also that $\alpha \in(-1,1)^{\mathrm{N}-1}$ is arbitrary. Consequently, (4) associates a family of continuous functions with each fixed $f \in c(\mathrm{I})$. The degrees of freedom offered by this procedure may be useful when some problems combined with approximation and optimizations have to be approached. Assume that the continuous function $\mathrm{b}$ depends linearly on $\mathrm{f}$, say for instance, $\mathrm{b}=\mathrm{Lf}$, where $L: c(\mathrm{I}) \rightarrow \mathrm{c}(\mathrm{I})$ is a linear and bounded operator with respect to the uniform norm or $L^{p}$ norm on C(I). Then the map $F^{\alpha}: c(\mathrm{I}) \rightarrow \mathrm{c}(\mathrm{I}), \mathrm{F}^{\alpha}(\mathrm{f})=\mathrm{f}^{\alpha}$ defines a linear operator referred to as an $\alpha$-fractal operator. For various properties of this map, its extension to $L^{p}$ spaces, and related developments, the reader is urged to refer researches f Navascu'es and coworkers scattered across the literature. However, here let us note the following: (i) the diversity option obtained due to the presence of parameters can be explored to choose best in a problem of approximation combined with optimization, (ii) the perturbation methodology described may serve to modify or preserve the properties of $f$, for instance, to reduce the order of regularity of the classical approximant, (iii) the procedure provides new geometric possibilities, that is, in the non-smooth case, the graph owns a fractal dimension and this parameter provides an index for experimental signals.

Let us conclude this short article by hinting at the applications of fractal functions. Having provided the above brief introduction to fractal interpolation and its interconnection with classical approximants, it is not difficult to find applications of FIFs in almost every field wherein information available in finite number of grid points has to be modeled with a continuous function. The realm of applications includes geometric design, data visualization, reverse engineering, physics and chemistry, image compression, signal processing, and wavelet theory. The reason for this variety of applications lies in the underlying complicated mathematical structure of fractal functions, produced with simple recursive construction. It has been noted that for certain problems, they provide better approximants than their classical nonrecursive counterparts. For a specific application, let us mention that in reference [21], fractal interpolation of electroencephalographic recordings is used to describe the increase in the bioelectric complexity during some tests of attention in children and to compute other electroencephalographic parameters. Many areas of fractal functions and their applications are yet to be explored, for instance, calculating Hausdorff dimension of a general FIF is a challenging open problem.

We strongly believe that the field of fractal functions has bright future, however to be able to contribute to it, the reader should leave the idea of staying in the comfort of well-known classical approximation theory and start enjoying the benefits of the more versatile fractal approximants, to supplement the former if not to replace.

\section{References}

1. Barnsley MF (1986) Fractal functions and interpolation. Constr Approx 2: 303329 .

2. Barnsley MF (1988) Fractals Everywhere, Academic Press, Orlando, Florida. 
Citation: Navascues MA, Chand AKB, Viswanathan P, Sebastan MV (2014) Fractal Functions in Interpolation and Approximation: A Bird's-Eye View. J Appl Computat Math 3: 188. doi:10.4172/2168-9679.1000188

Page 3 of 3

3. Barnsley MF, Harrington AN (1989) The calculus of fractal interpolation functions. J Approx Theory 57: 14-34.

4. Barnsley MF, Elton, Hardin D, Massopust $P$ (1989) Hidden variable fractal interpolation functions. SIAM J Math Anal 20: 1218-1242.

5. Barnsley MF, Vince A (2011) The chaos game on a general iterated function system. Ergod Th and Dynam Sys 31: 1073-1079.

6. Berger MA (1992) Random affine iterated function systems: curve generation and wavelets. SIAM Review 34: 361-385.

7. Bouboulis $P$, Dalla $L$ (2007) A general construction of fractal interpolation functions on grids of Rn. European J Appl Math 18: 449-476.

8. Chand AKB, Kapoor GP (2008) Stability of affine coalescence hidden variable fractal interpolation functions. Nonlinear Anal TMA 68: 3757-3770.

9. Chand AKB, Navascues MA (2008) Natural bicubic spline fractal interpolation. Nonlinear Anal TMA 69: 3679-3691.

10. Chand AKB, Viswanathan P (2013) A constructive approach to cubic Hermite fractal interpolation function and its constrained aspects, BIT Numer Math 53 : 841-865.

11. Chen S (1999) The non-differentiability of a class of fractal interpolation functions, Acta Math Sci 19: 425-430.

12. Dalla L, Drakopoulos V (1999) On the parameter identification problem in the plane and polar fractal interpolation functions, J. Approx. Theory 101: 289-302.

13. Gang C (1996) The smoothness and dimension of fractal interpolation functions. Appl Math-JCU 11: 409-418.
14. Hutchinson JE (1981) Fractals and self similarity. Indiana Univ Math J 30: 713747.

15. Li J, Su W (2014) The smoothness of fractal interpolation functions on R and on p-series local fields, Discrete Dyn.

16. Massopust PR (1990) Fractal surfaces. J Math Anal Appl 151: 275-290.

17. Navascues MA (2005) Fractal polynomial interpolation. Z Anal Anwend 25 401-418.

18. Navascues MA, Fractal trigonometric approximation. Electron Trans Numer Anal 20: 64-74.

19. Navascues MA, Sebastian MV (2003) Some results of convergence of cubic spline fractal interpolation functions. Fractals 11: 1-7.

20. Navascues MA, Sebastian MV (2004) Generalization of Hermite functions by fractal interpolation. J Approx Theory 131: 19-29.

21. Navascues MA, Sebastian MV (2004) in: Thinking in Patterns: Fractals and Related Phenomena in Nature, Novak M.M.(ed.), World Sci.

22. Viswanathan $P$, Chand AKB (2014) Fractal rational functions and their approximation properties. J Approx Theory 185: 31-50.

23. Viswanathan P, Chand AKB, Navascues MA (2014) Fractal perturbation preserving fundamental shapes: bounds on the scale factors. J Math Anal App 419: 804-817.

24. Wang HY, Yu JS (2013) Fractal interpolation functions with variable parameters and their analytical properties, J Approx Theory 175: 1-18.

25. Xie H, Sun $H$ (1997) The study of bivariate fractal interpolation functions and creation of fractal interpolation Surfaces. Fractals 5: 625-634. 